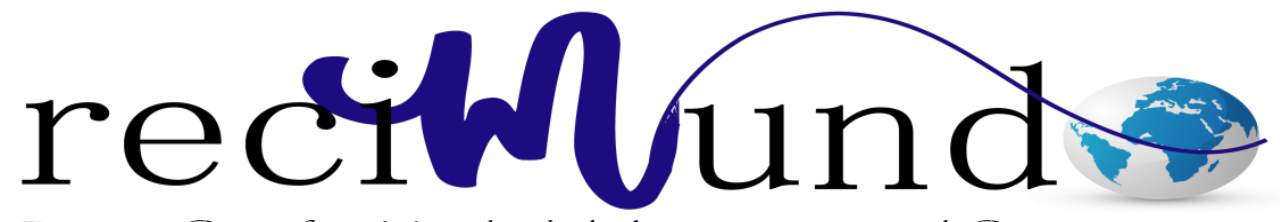

Revista Científica Mundo de la Investigación y el Conocimiento

Nancy Azucena Sorroza Rojas ${ }^{\text {a }}$ Jean Pool Jinez Sorroza ${ }^{\text {b }}$ Jesús Eliecer Rodríguez Villacis ${ }^{c}$; Washington Antonio Caraguay Ambuludi ${ }^{\mathrm{d}}$; Marcos Vinicio Sotomayor Sánchez ${ }^{\mathrm{e}}$

Las Tic y la resistencia al cambio en la Educación Superior

Revista Científica Mundo de la Investigación y el Conocimiento. Vol. 2 núm.2, mayo, ISSN: 2588-073X, 2018, pp. 477-495

DOI: 10.26820/recimundo/2.(2).2018.477-495

Editorial Saberes del Conocimiento

Recibido: 05/12/2017

Aceptado: 15/02/2018
a. Universidad Espirito Santo; nancysorroza@uees.edu.ec
b. Universidad Espirito Santo; jiimenez@ uees.edu.ec
c. Universidad Espirito Santo; jesusrodriguez@uees-edu.ec
d. Universidad Espirito Santo; wcaraguay@uees.edu.ec
e. Universidad Espirito Santo; mvinicio@uees.edu.ec 


\section{Las Tic y la resistencia al cambio en la Educación Superior}

Vol. 2, núm. 2., (2018)

Nancy Azucena Sorroza Rojas; Jean Pool Jinez Sorroza; Jesus Eliecer Rodríguez Villacis;

Washington Antonio Caraguay Ambuludi; Marcos Vinicio Sotomayor Sánchez

\section{RESUMEN}

El presente trabajo tiene como propósito reflexionar acerca de la incidencia de la resistencia al cambio en como obstáculo para que sea implantada exitosamente las TIC en la educación superior. Se explora diferentes concepciones acerca del cambio social en sus múltiples dimensiones (macro, meso y micro) partiendo de la premisa teórica de que la resistencia al cambio es parte del propio cambio en unidad dialéctica. Seguidamente se explora algunas de las teorías relevantes acerca del fenómeno de la resistencia al cambio, igualmente en su multidimensionalidad, para con ello revisar algunas investigaciones empíricas en el sector universitario de países latinoamericanos, para finalmente concluir de que la resistencia al cambio respecto a las TIC se evidencia fuertemente en sus docentes y estudiantes, pero también en la organización universitaria que no se ha comprometido a dar el impulso de los cambios organizacionales y culturales necesarios para que de manera significativa impacte las nuevas TIC en la formación de los ciudadanos, técnicos y profesionales que la sociedad del conocimiento está demandando.

Palabras clave: Cambio, resistencia al cambio, TIC, educación superior. 


\title{
Las Tic y la resistencia al cambio en la Educación Superior
}

Vol. 2, núm. 2., (2018)

Nancy Azucena Sorroza Rojas; Jean Pool Jinez Sorroza; Jesus Eliecer Rodríguez Villacis;

Washington Antonio Caraguay Ambuludi; Marcos Vinicio Sotomayor Sánchez

\begin{abstract}
The purpose of this paper is to reflect on the incidence of resistance to change as an obstacle to successful implementation of ICT in higher education. It explores different conceptions of social change in its multiple dimensions (macro, meso and micro) based on the theoretical premise that resistance to change is part of the change itself in dialectical unity. Then we explore some of the relevant theories about the phenomenon of resistance to change, also in its multidimensionality, to review some empirical research in the university sector of Latin American countries, to finally conclude that the resistance to change regarding ICT it is strongly evidenced in its teachers and students, but also in the university organization that has not committed to give the impetus of the organizational and cultural changes necessary for it to significantly impact the new ICT in the training of citizens, technicians and professionals that the knowledge society is demanding.
\end{abstract}

Key words: Change, resistance to change, ICT, higher education. 


\section{Las Tic y la resistencia al cambio en la Educación Superior}

Vol. 2, núm. 2., (2018)

Nancy Azucena Sorroza Rojas; Jean Pool Jinez Sorroza; Jesus Eliecer Rodríguez Villacis; Washington Antonio Caraguay Ambuludi; Marcos Vinicio Sotomayor Sánchez

\section{Introducción.}

El presente trabajo parte de la constatación vivencial de que los docentes universitarios, aun a finales de la segunda década del siglo XXI se resisten, en general al uso de las herramientas desarrolladas de las Tecnologías de la Información y la Comunicación (TIC) en los procesos de enseñanza aprendizaje.

Tan es así que en cursos de postgrado, e incluso doctorado, en los cuales han sido habilitadas plataformas Moodle de enseñanza aprendizaje en ambiente virtual, su uso tiende a ser realizado por una muy pequeña minoría, mientras que los muchos sólo se atreven al uso de correos electrónicos.

Tal situación por cierto no es nueva. Cuando en los desarrollos tecnológicos de la escritura mecanizada se daba el paso de la escritura en máquinas de escribir mecánicas, no pocas secretarias se negaban a dar el paso hacia la utilización de las máquinas eléctricas y mucho menos la de las electrónicas.

Situación similar encontramos con el uso de los paquetes de ofimática. Aquellos que se han posicionado con mayor fuerza son aquellos vinculados a Microsoft en tanto software propietario, especialmente y en sus utilidades más simples, el procesador de palabras (Word), el manejador de bases de datos (Excel) y el programa para presentaciones (PowerPoint). ..Mientras que las iniciativas de software libre, con la pretensión de liberar la dependencia del conocimiento y la utilidad respecto al software propietario, no encuentra acogida en el personal de las organizaciones, incluso en la administración pública y las universidades en las que se han adelantado normativas para su implantación. No obstante las ventajas del software libre, 


\section{Las Tic y la resistencia al cambio en la Educación Superior}

Vol. 2, núm. 2., (2018)

Nancy Azucena Sorroza Rojas; Jean Pool Jinez Sorroza; Jesus Eliecer Rodríguez Villacis; Washington Antonio Caraguay Ambuludi; Marcos Vinicio Sotomayor Sánchez

específicamente su resistencia a ser corrompidos por virus informáticos y a ser hackeados, su uso es evidentemente minoritario.

Son estas constataciones empíricas de un fenómeno sumamente extendido que es conocido como resistencia al cambio, con su expresión específica denominada resistencia tecnológica.

En el desarrollo del artículo, se explora diferentes concepciones acerca del cambio social en sus múltiples dimensiones (macro, meso y micro) partiendo de la premisa teórica de que la resistencia al cambio es parte del propio cambio en unidad dialéctica. Seguidamente se rastrea algunas de las teorías relevantes acerca del fenómeno de la resistencia al cambio, igualmente en su multidimensionalidad, para con ello revisar algunas investigaciones empíricas en el sector universitario de países latinoamericanos, para finalmente concluir de que la resistencia al cambio respecto a las TIC se evidencia fuertemente en sus docentes y estudiantes, pero también en la organización universitaria que no se ha comprometido a dar el impulso de los cambios organizacionales y culturales necesarios para que de manera significativa impacte las nuevas TIC en la formación de los ciudadanos, técnicos y profesionales que la sociedad del conocimiento está demandando.

\section{Cambio y resistencia al cambio:}

Al intentar explicar en qué consiste el cambio (en un nivel macro), en específico el cambio social Guiddens (1998, p. 656) lo refiere como "alteraciones en la estructura subyacente de un objeto o de una situación durante un período de tiempo", mientras que Macionis \& Plummer (1999) lo refiere como "transformación de la cultura y de las instituciones sociales a lo 


\section{Las Tic y la resistencia al cambio en la Educación Superior}

Vol. 2, núm. 2., (2018)

Nancy Azucena Sorroza Rojas; Jean Pool Jinez Sorroza; Jesus Eliecer Rodríguez Villacis; Washington Antonio Caraguay Ambuludi; Marcos Vinicio Sotomayor Sánchez

largo del tiempo" (p. 640) el cual generalmente sería producido por la invención, el descubrimiento o la difusión, y el conflicto social. En general en sociología tiende a aceptarse como referente fundamental de la noción de cambio social la proveniente del pensamiento dialéctico de Heráclito, quien habría planteado que lo único permanente en el universo es el cambio, por tanto la condición normal es la constante transformación de todo. Para su explicación y a manera de premisa se tienen las principales leyes de la dialéctica: la ley de la unidad de contrarios o ley de las contradicciones y la ley del salto de lo cuantitativo a lo cualitativo. En la primera de ellas se asume que todo ente en el universo está constituido por la unidad contradictoria de aspectos o fuerzas internas, que se enfrentan permanentemente entre sí quedando una de ellas latente y la otra manifiesta; tal contradicción sería la fuente fundamental que impulsa el cambio, el cual se explica mediante la otra de las leyes, que lo describe como de permanente crecimiento (transformaciones incrementales) que se acumulan hasta su saturación, generándose las condiciones para que se produzcan saltos cualitativos o transformaciones radicales.

El evolucionismo, que se encuentra explícito o implícito en gran parte de las teorías sociológicas y antropológicas, nos describe el cambio, y dentro de él el cambio cultural, como un proceso contínuo de variación desde estadios muy simples por estadios cada vez más complejos y diferenciados. Una expresión más concreta de esta mirada evolucionista se encuentra en las teorías de la modernización, el progreso y el desarrollo.

En el ámbito meso social nos encontramos con definiciones del cambio específicamente en los colectivos humanos, con especial énfasis en las organizaciones, entendido como cambio organizacional. Es allí donde se ha posicionado la teoría o modelo de cambio organizacional de 


\section{Las Tic y la resistencia al cambio en la Educación Superior}

Vol. 2, núm. 2., (2018)

Nancy Azucena Sorroza Rojas; Jean Pool Jinez Sorroza; Jesus Eliecer Rodríguez Villacis;

Washington Antonio Caraguay Ambuludi; Marcos Vinicio Sotomayor Sánchez

Kurt Lewin, citado por Palazón (2016), quien desde una propuesta de cambio planificado diseñado bajo la disciplina de la Psicología social, "considera el cambio de comportamientos como el resultado de un conjunto de fuerzas dentro de un sistema", de las cuales algunas impulsan el cambio y otras la resistencia a este, produciéndose estados de equilibrio de fuerzas (dinámica de grupos/zona de confort) cuya ruptura a favor del cambio se produce al aumentarse la magnitud de la presión positiva y se disminuye la magnitud de aquella que lo obstaculiza. En tal contexto, se entendería el cambio como "introducir la nueva forma de trabajar, para crear esa cultura, nuevas pautas, nuevos comportamientos, interiorizar nuevas pautas".

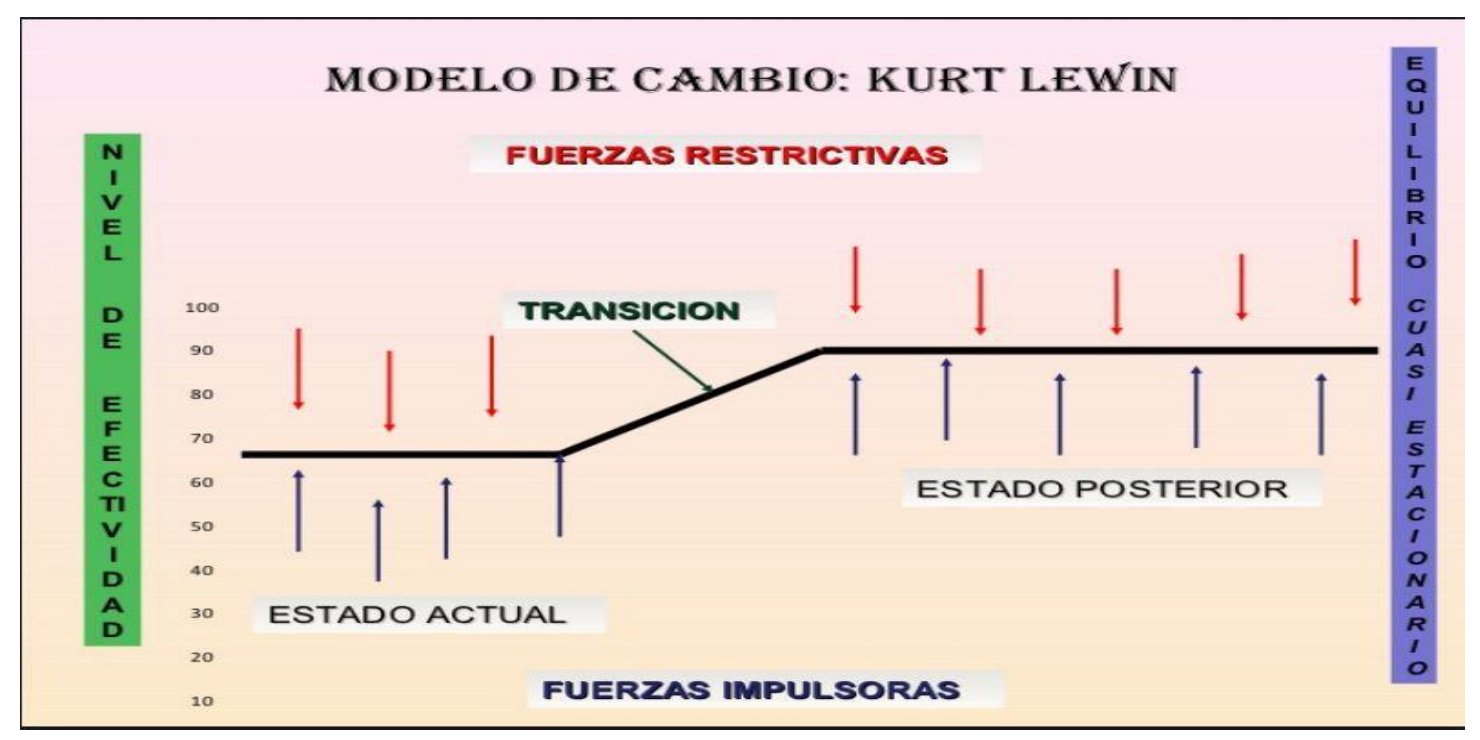

Luego de revisar múltiples concepciones y teorías acerca del cambio organizacional, Acosta (2002) termina asumiendo el enfoque de sistemas de Katz y Kahn (1983), según el cual las organizaciones cambian internamente, se transforman en correspondencia con los constantes cambios ambientales que las constriñen, generalmente vinculados con los cambios de carácter tecnológico: "el cambio organizacional corresponde a la respuesta que normalmente deben dar las organizaciones a aquellos ciclos de desarrollo que tiene el entorno" (Acosta , 2002, p. 13), 


\section{Las Tic y la resistencia al cambio en la Educación Superior}

Vol. 2, núm. 2., (2018)

Nancy Azucena Sorroza Rojas; Jean Pool Jinez Sorroza; Jesus Eliecer Rodríguez Villacis; Washington Antonio Caraguay Ambuludi; Marcos Vinicio Sotomayor Sánchez

visto con mirada evolucionista, donde aquellas organizaciones que no se adecúen tenderán a no sobrevivir en el tiempo, en el marco de la necesaria competencia con otras organizaciones, en una especie de "oposición de contrarios" con respecto a las demás organizaciones y con respecto al entorno. Así, Acosta (2002) termina definiendo el cambio organizacional como: el conjunto de transformaciones que se realizan en las distintas dimensiones de las organizaciones, es producido tanto por fuerzas naturales como impulsado por la voluntad de quienes las crean y las impulsan" (p. 14). De lo que se extrae coincidencias con los anteriores enfoques mencionados, pero destaca la coincidencia con Lewin al ubicar el cambio organizacional en el terreno del campo de fuerzas enfrentadas, es decir visión dialéctica, en la cual se identifican la fuerza o poder de la dirección organizacional que pretende darle orientación y direccionalidad a los cambios, la velocidad permitida por la inercia, lo que significaría que "aunque las directivas tengan el suficiente poder para instaurar los cambios existirán fuerzas que opondrán un nivel de resistencia directamente proporcional al poder de influencia que tengan sobre los grupos internos.” (p. 15)

Dado que se ha identificado el papel central de la tecnología en el terreno del cambio, tanto social como organizacional, e incluso a nivel micro social, destacamos el enfoque "neoshumpetereano" propuesto por Pérez (2010) en su disertación acerca del cambio técnico en su interacción con el contexto histórico. En este sentido, la autora coloca la innovación en el centro de los procesos de transformación tecno productivos que, en formas de ondas, se inician con la invención o creación científico técnica (lo técnicamente posible), para pasar por el filtro de la lógica económica (rentabilidad) que determina la posibilidad de que lo inventado se convierta en producto que vaya a los espacios de intercambio (lo económicamente viable) y que al expandirse 


\section{Las Tic y la resistencia al cambio en la Educación Superior}

Vol. 2, núm. 2., (2018)

Nancy Azucena Sorroza Rojas; Jean Pool Jinez Sorroza; Jesus Eliecer Rodríguez Villacis; Washington Antonio Caraguay Ambuludi; Marcos Vinicio Sotomayor Sánchez

e incidir en las prácticas y el sentido común (lo socialmente aceptable), terminan traduciéndose en cambios socio institucionales.

Shellemberg (1985) citado por Acosta (2002) describe con especial fluidez y especialización la propuesta de Lewin que explica el cambio grupal en su relación con el cambio individual. Tal cambio se produce en medio de tensiones entre las fuerzas propias del individuo, derivadas de sus necesidades individuales, y las que llama fuerzas inducidas provenientes del ambiente. De allí que tal modelo, que es el de mayor reconocimiento por la psicología social, el cambio individual estará en relación directa con el nivel de cohesión del grupo u organización, en cuyo alto nivel las metas individuales tenderán a no diferenciarse de las metas grupales, lo que facilitaría el mayor grado de facilidad de inducir cambios desde lo grupal y no desde lo individual.

Todo parece indicar que es más sencillo cambiar a los individuos integrados en un grupo que cambiar a cualquiera de ellos por separado. En tanto que los valores de grupo permanecen inalterables, el individuo se resistirá al cambio con más fuerza a medida que la distancia de los estándares de grupo sea mayor. Si se cambia el propio estándar del grupo, se elimina la resistencia debida a la relación entre el individuo y el grupo (Acosta, 2002, p. 86)

\section{La resistencia al cambio:}

De acuerdo a lo compartido por García y Rangel (2001), citando a Salinas (1975), la resistencia al cambio sería: 


\section{Las Tic y la resistencia al cambio en la Educación Superior}

Vol. 2, núm. 2., (2018)

Nancy Azucena Sorroza Rojas; Jean Pool Jinez Sorroza; Jesus Eliecer Rodríguez Villacis; Washington Antonio Caraguay Ambuludi; Marcos Vinicio Sotomayor Sánchez

El conjunto de reacciones disímiles por parte de los diferentes miembros de un mismo grupo que realizan de forma consciente e inconsciente, producto de una evaluación por parte de los mismos acerca de los efectos que cualquier reforma pueda tener sobre sus conveniencias personales (p. 74)

Se trata entonces de reacciones naturales, pues la resistencia es dialécticamente parte misma del proceso de cambio. Tales reacciones son entendidas como fuerzas que se oponen al avance de los cambios y transformaciones, y que generan equilibrios organizacionales en la búsqueda del mantenimiento de cierta estabilidad y en algunos casos de retorno a situaciones anteriores. Individuos y organizaciones se resisten al cambio lo cual obstaculiza la adaptación y el progreso.

Las fuentes o factores de la resistencia al cambio pueden ser de carácter individual u organizacional, según lo establece Robbins (1999). Los primeros se vinculan a las percepciones, personalidades y necesidades básicas individuales, tal como la necesidad de seguridad planteada en la jerarquía de las necesidades propuesta por Abrahan Maslow. Así mismo, se desprende tal resistencia individual a los hábitos, los temores a lo desconocido, la sensación de amenaza e inseguridad, el procesamiento selectivo de la información. Los segundos se desprenden de la naturaleza intrínsecamente conservadora de las propias organizaciones, expresado en: "inercia estructural, enfoque limitado del cambio, inercia al grupo, amenaza a la habilidad, amenaza a las relaciones ya establecidas de poder y amenaza a las asignaciones de recursos ya establecidas" (García y Rangel, 2001, p. 75) 


\section{Las Tic y la resistencia al cambio en la Educación Superior}

Vol. 2, núm. 2., (2018)

Nancy Azucena Sorroza Rojas; Jean Pool Jinez Sorroza; Jesus Eliecer Rodríguez Villacis; Washington Antonio Caraguay Ambuludi; Marcos Vinicio Sotomayor Sánchez

Identifica Domínguez (2013), citando a Kurt Lewin (1951), la clasificación de la resistencia en cuatro tipos:

- $\quad$ Abierta: aquella que tiene carácter franco y frontal.

- Implícita: aquella que no se manifiesta directamente

- $\quad$ Inmediata: la que se muestra como reacción de muy corto plazo.

- $\quad$ Diferida: aquella que se proyecta hacia el futuro

En este orden de ideas la Organización Mundial del Movimiento Scout Región Interamericana $(\mathrm{S} / \mathrm{F})$ citan a Kreitner y Kichini con su propuesta de contínuo de resistencia al cambio, en la cual identifica las situaciones extremas con sus respectivas facetas intermedias. Las situaciones extremas serían por un lado como máxima expresión de resistencia al cambio el sabotaje deliberado como parte de la resistencia activa hasta el extremo opuesto en la faceta de la aceptación con el entusiasmo manifiesto. "El continuo muestra que la resistencia puede ser tan sutil como la resignación pasiva y tan patente como un sabotaje deliberado" (p. 5) 


\section{Las Tic y la resistencia al cambio en la Educación Superior}

Vol. 2, núm. 2., (2018)

Nancy Azucena Sorroza Rojas; Jean Pool Jinez Sorroza; Jesus Eliecer Rodríguez Villacis; Washington Antonio Caraguay Ambuludi; Marcos Vinicio Sotomayor Sánchez

Continuo de resistencia al cambio según Robert

Kreitner y Angelo Kinichi, Arizona State University.

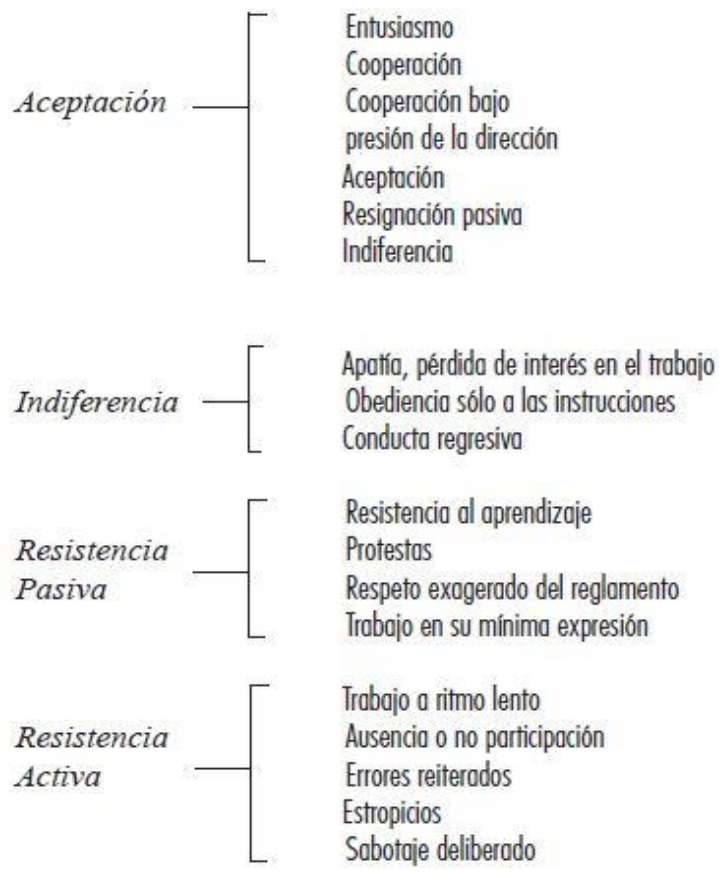

Fuente: Organización Mundial del Movimiento Scout Región Interamericana (S/F. p.5)

Mientras que, por su parte Pérez (2010) apunta que:

El proceso de incorporación de los modelos tecnológicos y organizacionales más efectivos en las instituciones públicas ha sido lento y dista de estar plenamente establecido. Esto no debe sorprender. La inercia organizativa es un fenómeno humano y social de resistencia al cambio bien conocido. (...)Históricamente, entonces, éstas han estado considerablemente rezagadas (típicamente por 20 a 30 años) y sólo han imitado los principios paradigmáticos desarrollados en las empresas cuando se han visto forzadas a responder a las presiones políticas 


\section{Las Tic y la resistencia al cambio en la Educación Superior}

Vol. 2, núm. 2., (2018)

Nancy Azucena Sorroza Rojas; Jean Pool Jinez Sorroza; Jesus Eliecer Rodríguez Villacis; Washington Antonio Caraguay Ambuludi; Marcos Vinicio Sotomayor Sánchez

que exigen efectividad. (...)La fuerte resistencia resultante requiere mecanismos aún más fuertes

para inducir el cambio. Es la generación más joven, la que nunca aprendió las prácticas del paradigma anterior, la que adopta y aplica los nuevos principios más naturalmente. (p. 15/16).

La resistencia al cambio en la educación superior y las TIC

Las diferentes manifestaciones de resistencia al cambio que teóricamente se han descrito,

en sus clasificaciones y formas han sido de alguna manera identificados en estudios empíricos en diferentes países latinoamericanos. Por ejemplo, Ríos y González (2016) reseñan su investigación cuyo objeto fue estudiar desde una metodología cualitativa, de Historias de Vida, la resistencia a las TIC por parte de los docentes de una universidad colombiana. Luego de analizar los datos recopilados en las entrevistas a profundidad realizadas a docentes de dicha universidad emergieron del desvelamiento de sus discursos una serie de categorías, a saber: Resistencia, desidia, incertidumbre, temor y confort. La primera en emerger fue la incertidumbre acompañada de fatiga respecto a las actividades cotidianas en el aula de clase y en su interacción con los estudiantes. Mientras que se denota que el sistema simbólico de los docentes queda confrontado en el proceso de enseñanza aprendizaje con el de los jóvenes, para quienes las TIC serían parte naturalizada de su vida. Así sucesivamente fueron emergiendo las mentadas categorías, hasta que surgió una que marca seriamente la resistencia al cambio individual como lo es el estilo de vida o zona de confort, entendiendo que 


\section{Las Tic y la resistencia al cambio en la Educación Superior}

Vol. 2, núm. 2., (2018)

Nancy Azucena Sorroza Rojas; Jean Pool Jinez Sorroza; Jesus Eliecer Rodríguez Villacis; Washington Antonio Caraguay Ambuludi; Marcos Vinicio Sotomayor Sánchez

La zona en la que se asumen comportamientos, conocimientos, rutinas, habilidades, actitudes, reacciones, hábitos y vivencias. Todo aquello conocido y a lo que se está acostumbrado, pero es claro que para todas las personas este estado no necesariamente significa agrado; es posible pensar que este tipo de estado solo puede generar tranquilidad, pues es el espacio que se conoce, que se vivencia, donde se moviliza la persona. Es decir, la expresión individual más patente de la resistencia al cambio.

Otra investigación empírica se realizó en la Universidad Autónoma de Nuevo León (UANL) en México, quienes describen a profundidad la problemática de la resistencia al cambio tecnológico en una universidad mexicana, a partir del estudio de tres actores fundamentales, dos de carácter individual ( docentes y estudiantes) y otro de carácter colectivo como lo es el sistema educativo, obteniendo como conclusión que los docentes y estudiantes manifestaban diferentes expresiones de resistencia al cambio tecnológico, a saber: "para el estudiante: su incapacidad de ver a la tecnología como una herramienta para aprehender sino solo para divertirse; para el maestro el ver la tecnología como un instrumento de terror que lo saque de su zona de confort" (Iglesias \& Ávila, 2012, p.6)

Mientras que el reclamo para "el sistema educativo: centrar su actuar en actividades burocráticas y no en lograr superar o resolver las disposiciones que impidan la Enseñanza y/o el Aprendizaje del estudiante. (Iglesias \& Ávila, 2012, p.6) 


\section{Las Tic y la resistencia al cambio en la Educación Superior}

Vol. 2, núm. 2., (2018)

Nancy Azucena Sorroza Rojas; Jean Pool Jinez Sorroza; Jesus Eliecer Rodríguez Villacis; Washington Antonio Caraguay Ambuludi; Marcos Vinicio Sotomayor Sánchez

Otra investigación, en la búsqueda de determinar la percepción, uso y apropiación de los

docentes universitarios del nivel pregrado respecto a las TIC, destacan entre el logro de sus indagaciones el determinar: la minusvalía en que se perciben los docentes comparándose con sus alumnos en cuanto al manejo de las nuevas TIC, que su aplicación implica la inversión de mayor cantidad de tiempo en preparación de clases, los docentes subutilizan las herramientas informáticas tendiendo a dar sólo uso a las herramientas ofimáticas: "presentación de videos para ejemplificar el tema de estudio, la presentación de contenidos en Power point" (Salazar, 2011, p. 47), cerca del $50 \%$ de los docentes se consideran a sí mismos como rezagados en su incorporación al manejo de las nuevas TIC, mientras que sólo un 25\%, generalmente los más jóvenes, podrían considerarse pioneros y son quienes hacen mejor apropiación de las tecnologías. La universidad no ha adecuado los procesos para que los docentes perciban que vale la pena la incorporación de las TIC en su dinámica regular de enseñanza aprendizaje, las plataformas de educación a distancia mediadas por TIC no son tan populares ni entre los docentes ni entre los estudiantes, quienes prefieren el uso de las redes sociales si requieren publicar contenidos.

Finalmente, reseñamos una investigación realizada en Venezuela, cuyo propósito fue establecer las necesidades de formación docente para la exitosa incorporación de las TICs en la enseñanza universitaria. En este sentido, entre otras cosas destaca que estudios a nivel internacional evidenciarían el atraso de los docentes en el empleo de las nuevas TIC en comparación con otros 


\section{Las Tic y la resistencia al cambio en la Educación Superior}

Vol. 2, núm. 2., (2018)

Nancy Azucena Sorroza Rojas; Jean Pool Jinez Sorroza; Jesus Eliecer Rodríguez Villacis; Washington Antonio Caraguay Ambuludi; Marcos Vinicio Sotomayor Sánchez

ámbitos de la vida social. Así mismo, se subraya que los docentes valoran positivamente el uso de las TIC por los estudiantes, no así para ellos mismos, dado que no percibirían las ventajas a sus prácticas. Reseña adicionalmente la identificación de factores que incidirían en la renuencia del docente al uso de TIC en el aula, específicamente: "la inadecuada formación y capacitación del educador en el manejo de los recursos tecnológicos, las políticas educativas laxas en el área, las inseguridades o los miedos de los usuarios asociado al fenómeno denominado "tecnofobia"”. (Amaya \& Amaya, 2010, p.p. 254). Destaca entre las conclusiones la identificación de nudos problemáticos más vinculado a la universidad como organización que a los docentes en tanto agentes individuales que reflejan sus propias resistencias al cambio. Siendo así enfatiza la investigación en que la incorporación plena de las TIC a los procesos de enseñanza universitaria: Debe estar apoyada en un proyecto educativo que contemple las concepciones pedagógicas en las que el docente sustentará su práctica y abarcar tanto una representación del marco de trabajo escolar más inmediato, en el que la utilización didáctica de las TIC llegan a adquirir unos significados diferentes a los que tiene la interacción didáctica sin ellos, como una visión global que rediseñe las reglas del juego de la sociedad en su interacción con tales medios (Amaya \&Amaya, 2010, p. 258) 


\section{Las Tic y la resistencia al cambio en la Educación Superior}

Vol. 2, núm. 2., (2018)

Nancy Azucena Sorroza Rojas; Jean Pool Jinez Sorroza; Jesus Eliecer Rodríguez Villacis;

Washington Antonio Caraguay Ambuludi; Marcos Vinicio Sotomayor Sánchez

\section{Conclusión.}

A manera de conclusión podemos afirmar que:

1. Existe evidencia de resistencia al cambio tanto de los docentes como individuos como de la universidad como organización.

2. Las TIC avanzan en su desarrollo (innovación) a una velocidad muy superior a la que son asimiladas por las organizaciones de educación universitaria así como por sus docentes. De manera tal que no se puede decir que haya impactado en tanto cambio socio institucional con la suficiente fuerza en la educación superior, manteniéndose las plataformas educativas basadas en TIC en un plano muy rezagado en comparación con la enseñanza tradicional.

3. Se evidencia resistencia al cambio respecto a las TIC en cada una de las facetas, factores y formas identificadas por la teoría, tanto en el plano individual de los docentes universitarios como en el plano organizacional de la universidad.

4. No se observa en el liderazgo de la educación superior un compromiso con el impulso de los cambios organizacionales y culturales necesarios para que de manera significativa impacte las nuevas TIC en la formación de los ciudadanos, técnicos y profesionales que la sociedad del conocimiento está demandando. 


\section{Las Tic y la resistencia al cambio en la Educación Superior}

Vol. 2, núm. 2., (2018)

Nancy Azucena Sorroza Rojas; Jean Pool Jinez Sorroza; Jesus Eliecer Rodríguez Villacis;

Washington Antonio Caraguay Ambuludi; Marcos Vinicio Sotomayor Sánchez

\section{Bibliografía.}

Acosta, C. (2002) Cuatro preguntas para iniciarse en cambio organizacional. Revista Colombiana de Psicología, No 11, p.p. 9-24, Recuperado de : www.redalyc.org/pdf/804/80401101.pdf

Amaya, R y Amaya, Z. (2010) La formación docente: reto para la incorporación de las TIC a la enseñanza, Revista Ciencias de la Educación, Vol. 20, No 36, p.p. 247-260, recuperado de: servicio.bc.uc.edu.ve/educacion/revista/n36/art13.pdf

Guiddens, A. (1998) Sociología. Alianza Editorial. Madrid, España

Iglesias, M. y Ávila, L. (2012) La resistencia al cambio dentro del proceso de enseñanzaaprendizaje centrada en tres actores sociales del mismo. Ponencia presentada al VIII Coloquio Internacional sobre Políticas Sectoriales: Reconfiguración de las políticas sociales en una sociedad desigual. Facultad de Trabajo Social y Desarrollo Humano de la UANL, Recuperado de: http://eprints.uanl.mx/8108/1/i1_1.pdf

López, G y Domínguez, J. (2013) Resistencia al cambio, factor de fracaso en las pequeñas y medianas empresas en la zona de Atlacomulco, (Tesis inédita de pregrado) Universidad Autónoma del Estado de México. Centro Universitario UAEM Atlacomulco. Recuperado de: eprints.uanl.mx/5280/1/1020148618.PDF

Macionis, J y Plummer K. (1999) Sociología. Prentice Hall, Madrid, España.

Organización Mundial del Movimiento Scout Región Interamericana (S/F) Cambio y Resistencia al Cambio. Fascículo 7 Serie Organizaciones Scouts abiertas al aprendizaje. Red de Desarrollo Institucional, Chile. Recuperado de: www.scout.org/sites/default/files/library_files/RDIfasciculo07.pdf

Palazón, A (29 diciembre 2016) La Teoría del cambio según Kurt Lewin. La gestión del cambio en positivo. Recuperado de: http://amandapalazon.com/la-teoria-del-cambio-segun-kurtlewin/

Pérez, C. (2010) Revoluciones tecnológicas y paradigmas tecno-económicos. Cambridge Journal of Economics, Vol. 34, $\mathrm{N}^{\mathrm{o}}$ 1, pp. 185-202. Recuperado de: www.carlotaperez.org/.../Revoluciones_tecnologicas_y_paradigmas_tecnoeconomicos..

Ríos, A. y González, L (2016) Resistencia a las TIC en docentes del Politécnico Colombiano Jaime Isaza Cadavid. Plumilla Educativa, $\mathrm{N}^{\mathrm{o}}$ 17, p.p. 1-15. Recuperado de: oaji.net/pdf.html?n=2017/5027-1496079980.pdf

Robbins, S. (1999). Comportamiento Organizacional. Octava Edición. Editorial Prentice Hall. México 


\section{Las Tic y la resistencia al cambio en la Educación Superior}

Vol. 2, núm. 2., (2018)

Nancy Azucena Sorroza Rojas; Jean Pool Jinez Sorroza; Jesus Eliecer Rodríguez Villacis;

Washington Antonio Caraguay Ambuludi; Marcos Vinicio Sotomayor Sánchez

Salazar, M (2011) ¿cómo ven los docentes las TIC? Percepciones, uso y apropiación de tic en los docentes de la facultad de comunicaciones. (Tesis inédita de pregrado) Universidad de Antioquia, Medellín, Colombia. 